\title{
Um Método Exato para o Problema do Caixeiro Viajante com Grupamentos Euclidiano e Simétrico
}

\author{
Mário Mestria, \\ Coordenadoria de Engenharia Elétrica, Campus Vitória, IFES \\ Avenida Vitória, 1729 - Bairro Jucutuquara \\ 29040-780, Vitória, ES \\ E-mail: mmestria@ifes.edu.br, mmestria@uol.com.br.
}

Resumo: Nesse artigo, é proposto um método exato para resolver o Problema do Caixeiro Viajante com Grupamentos (PCVG). O PCVG é uma generalização do Problema do Caixeiro Viajante (PCV), onde os vértices são particionados em grupos disjuntos e o objetivo é encontrar um ciclo hamiltoniano de custo mínimo tal que os vértices de cada grupo são visitados de forma contígua. A formulação para o método inclui a restrição de Chisman (1975) e uma formulação da literatura. Os testes foram realizados, através do software CPLEX Paralelo, em tipos de instâncias com diversas granularidades, com vértices e grupos de tamanhos variáveis. Resultados computacionais mostraram que o método encontrou diversas soluções ótimas e bons limites inferiores com tempo computacional relativamente baixo.

Palavras-chave: Método Exato, Formulação Matemática, Otimização Combinatória

\section{Introdução}

O PCVG foi proposto por [2] para resolver um problema real de roteamento automático em sistemas de armazenagem. Neste exemplo particular de sistemas de armazenagem, as ordens de despacho de produtos devem ser respeitadas e estas ordens contêm várias subordens, mais conhecidas como bills-of-lading. Este tipo de logística foi modelado como um PCVG, onde os vértices representam as localizações dos estoques dos produtos, as arestas representam as distâncias a serem percorridas pelo veículo motorizado e os grupos representam as bills-of-lading.

Limites inferiores utilizando Relaxação Lagrangeana foram desenvolvidos em [7] para o PCVG. O método para obtenção do limite inferior baseou-se na árvore geradora mínima desenvolvida primeiro para o PCV. Foram criados vários conjuntos de instâncias variando o número de vértices de 80 a 150 com diferentes números de grupos. Os valores médios dos limites inferiores alcançaram gap igual a $0,35 \%$.

Em [8] uma Busca Tabu combinada com uma fase de diversificação usando um Algoritmo Genético foi proposta para o PCVG, mas a ordem de visita dos grupos já era definida a priori. $\mathrm{O}$ algoritmo parte de um conjunto de soluções geradas aleatoriamente. A cada solução individual gerada aplica-se a Busca Tabu. Um Algoritmo Genético (AG) básico (sem componentes que realizam uma busca mais intensiva, tais como: reconexão de caminhos e/ou Variable Neighborhood Descent) foi proposto para o PCVG por [12]. O AG utilizou na reprodução o operador de seleção de classificação linear e a Recombinação de Arestas. O algoritmo tratou de forma independente o roteamento intergrupos e intragupos, dando prioridade a solução intergrupos e em seguida a solução intragrupos.

No artigo apresentado por [5] um outro AG, também básico, usa o critério de visitação dos vértices de cada grupo de forma aleatória sem estabelecer uma relação com as distâncias entre os vértices. A escolha da ordem de visita de cada grupo também é aleatória. Os algoritmos $\alpha$-aproximados para o PCVG com diferentes variantes são encontrados em [1] e [6]. A maioria destes algoritmos necessita estabelecer em cada grupo os vértices de entrada e de saída e uma ordem pré-definida de visita dos grupos. 
O objetivo deste artigo é propor um método exato para solucionar o PCVG na sua forma mais genérica, onde a ordem de visita dos grupos fica a cargo do próprio algoritmo exato. A estrutura do artigo é formada a seguir. A segunda seção mostra a definição formal para o PCVG, a terceira seção descreve a formulação proposta para o PCVG e a quarta seção mostra os resultados computacionais, comparações e discussões. Na última seção são mostradas as conclusões.

\section{Definição do Problema}

O Problema do Caixeiro Viajante com Grupamentos (PCVG) é uma extensão do Problema do Caixeiro Viajante (PCV), um dos problemas mais estudados na área de otimização combinatória. No caso particular do PCVG ter somente um grupo ou analogamente um vértice em cada grupo, o PCVG torna-se PCV. Neste sentido, o PCVG é também $\mathcal{N} \mathcal{P}$-difícil [6]. Uma descrição do PCVG na estrutura de um grafo é dado a seguir: seja $G=(V, A)$ um grafo completo, direcionado, simétrico e ponderado com um conjunto de vértices $V=\left\{v_{1}, v_{2}, \ldots, v_{n}\right\}$ e um conjunto de arcos $A=\left\{\left(v_{i}, v_{j}\right): v_{i}, v_{j} \in V, i \neq j\right\}$ em ambas as direções com mesmo peso. O conjunto de vértices $V$ é particionado em $m$ grupos $V_{1}, V_{2}, \ldots, V_{m}$, onde: $V=\bigcup_{i=1}^{m} V_{i}, \forall i$ e $V_{i} \cap V_{j}=\emptyset$ para todos $i$ e $j, i \neq j$. Assumindo que um custo não-negativo $c_{i j}$ é associado com o $\operatorname{arco}\left(v_{i}, v_{j}\right) \in A$, o PCVG consiste em determinar um ciclo hamiltoniano de custo mínimo em $G$, tal que os vértices de cada grupo são visitados contiguamente, denominado de PCVG não-orientado.

O custo $c_{i j}$ é a distância euclidiana que corresponde, neste trabalho, a distância geométrica $d_{i j}$ entre dois vértices $v_{i}$ e $v_{j}$ no plano bidimensional. Neste contexto, instâncias euclidianas significam que quando os métodos utilizam estas instâncias para resolução do PCVG fazem o uso das distâncias euclidianas. Visto que as instâncias abordadas neste trabalho são euclidianas e simétricas, a rigor temos o Problema do Caixeiro Viajante com Grupamentos Euclidiano e Simétrico.

\section{Formulação Matemática Proposta}

Diversas formulações do Problema do Caixeiro Viajante (PCV) podem ser encontradas em [11]. Somente duas destas formulações (a de [4] e a de [10]) podem ser adaptadas para o Problema do Caixeiro Viajante com Grupamento (PCVG) incorporando as restrições de [2]. Estas restrições mantêm os vértices dos grupos a serem visitados de uma forma contígua, que é justamente uma das definições para o PCVG. As duas formulações são denominadas aqui de Dantzig/Chisman e Miller/Chisman (descrita a seguir).

Na formulação de Dantzig/Chisman suas restrições crescem exponencialmente na proporção de $2^{n}$, enquanto que na formulação de Miller/Chisman suas restrições crescem na proporção polinomial $\left(n^{2}\right)$. Na formulação Dantzig/Chisman demanda um tempo maior para encontrar a solução ótima do que a formulação de Miller/Chisman. Neste trabalho, nos testes computacionais foi observado que usando a formulação Dantzig/Chisman o tempo passa de menos de um segundo para 351 segundos, quando varia-se o número de vértices da instância de 10 para 20. Já na formulação de Miller/Chisman, o tempo para encontrar a solução ótima é sempre menor que um segundo para este mesmo conjunto de instâncias. Ambas as formulações foram executadas e implementadas num mesmo ambiente de hardware e software descritos na seção 4.

A partir de instâncias com número de vértices maior que 20 não foi possível encontrar nenhuma solução no modelo de Dantzig/Chisman, devido não ser possível armazenar o problema na memória utilizando esta formulação. Desta forma, o método exato proposto utiliza a formulação de Miller/Chisman para encontrar soluções ao PCVG.

A seguir é mostrado a formulação Miller/Chisman. Esta formulação para o PCVG utiliza programação inteira 0-1. Seja um caixeiro viajante partindo da origem da cidade 1 sem perda de generalidade. A função objetivo 1 requer que se minimize o custo total percorrido pelo caixeiro viajante. As restrições 2 garantem que de cada cidade $i$ só se pode sair para uma única cidade 
$j$. As restrições 3 significam que para cada cidade $j$ há uma única origem em $i$. Estes dois conjuntos de restrições implicam que somente duas arestas podem ser conectadas a cada vértice formando um conjunto de ciclos possivelmente disjuntos. As restrições 4 garantem não haver subciclos desconexos da origem na solução.

$$
\begin{array}{cl}
\text { Min } & z=\sum_{i=1}^{n} \sum_{j=1}^{n} c_{i j} x_{i j} \\
\text { s.a. } & \forall i \in V \\
\sum_{j=1}^{n} x_{i j}=1, & \forall j \in V \\
\sum_{i=1}^{n} x_{i j}=1, & \\
u_{i}-u_{j}+(n-1) x_{i j} \leq(n-2), & 2 \leq i \neq j \leq n \\
\sum_{i \in V_{k}} \sum_{j \in V_{k}} x_{i j}=\left|V_{k}\right|-1, & \forall V_{k} \subset V,\left|V_{k}\right| \geq 1, k=1, \ldots, m \\
u_{i} \geq 0 & 2 \leq i \leq n \\
x_{i j} \in\{0,1\} & \forall i, j \in V
\end{array}
$$

As restrições 5 indicam que a soma das variáveis $x_{i j}$ na partição do grupo $V_{k}, k=1, \ldots, m$, sendo $m$ o número total de grupos, alcançam o valor da cardinalidade $\left|V_{k}\right|$ deste grupo menos 1, forçando em conjunto com as restrições 2 e 3, a formação de um caminho dentro deste grupo. As variáveis binárias $x_{i j}$ assumem o valor igual a 1 se a cidade $i$ for conectada a cidade $j$ na solução. Caso contrário, será igual a 0.

As restrições 4 garantem não haver subciclo na solução e que o caixeiro viajante parte da cidade de número 1 (vértice origem). As variáveis $u_{i}, i=2, \ldots, n$ representam a sequência das cidades $i$ a serem visitadas tendo a relação $u_{j}-u_{k} \leq-1, \forall j, k \in V$ sendo $k$ e $j$ cidades contíguas na solução, $x_{j k}=1$. Esta formulação envolve $n^{2}-n+2+m$ restrições e $n^{2}$ variáveis inteiras e $n-1$ variáveis contínuas.

Também é proposta a formulação 1 a 4 mais as restrições 6 e 7 com as arestas intergrupos penalizadas, denominada de Miller/Pen, para confrontar com a formulação Miller/Chisman.

\section{Resultados Computacionais utilizando a Formulação Matemática}

Nesta seção são descritos os resultados dos testes computacionais efetuados para a solução do PCVG. Serão mostrados resultados utilizando a formulação matemática da literatura incorporando as restrições de [2] e a formulação Miller/Pen.

Para avaliar e validar o método exato foram utilizadas as instâncias euclidianas de [9], disponíveis através de acesso em $<$ http://labic.ic.uff.br/Instance/index.php $>$. Estes conjunto de instâncias são aplicadas ao PCVG, em sua versão genérica.

Estas seis formas diferentes de instâncias contidas neste conjunto, sem fixação da ordem de visitas aos grupos para o PCVG, são instâncias euclidianas e simétricas. Os resultados computacionais apresentados, a seguir, foram obtidos para este conjunto de instâncias para verificar o desempenho do método exato para instâncias de pequeno, médio e grande porte. Foram utilizadas as formulações matemáticas proposta ao PCVG e o software CPLEX [3] com a finalidade de encontrar soluções ótimas para maior parte das instâncias consideradas.

Para as instâncias mostradas, a seguir, o CPLEX Paralelo, versão 11.2, foi executado num computador com 4 núcleos Intel Core 2 Quad, 2.83 GHz com 8 GB de RAM num sistema operacional Linux Ubuntu versão 4.3.2-1. A partir de agora o CPLEX Paralelo, versão 11.2, será mencionado simplesmente por CPLEX. Os modelos de Miller/Chisman e Miller/Pen possibilitaram encontrar soluções através do CPLEX sem ocorrer estouro de memória. Foram realizados testes computacionais a diversos tipos de instâncias utilizando ambos modelos: Miller/Chisman (sem penalizar as arestas intergrupos) e Miller/Pen (com as arestas intergrupos penalizadas). A seguir, é mostrada na Tabela 1 a comparação entre os tempos computacionais usando as formulações dos modelos Miller/Chisman e Miller/Pen demandado pelo CPLEX para diversos 
tipos de instâncias. Na primeira coluna da Tabela 1 é mostrado o número de vértices, na segunda o número de grupos, na terceira coluna as instâncias, na quarta coluna o tipo de instância e nas duas últimas colunas os tempos computacionais obtidos pelo CPLEX em segundos com a formulação sem penalizar as arestas inter-grupos e com a formulação que penaliza as arestas inter-grupos, respectivamente. Os valores alcançados pelo CPLEX na função objetivo foram iguais em ambas formulações para todas as instâncias e estão mostrados nas Tabelas 2, 3 e 4 .

\begin{tabular}{|c|c|c|c|c|c|}
\hline \#nós & $\# V_{i}$ & Instâncias & Tipo & Tempo sem Pen.(s) & Tempo com Pen.(s) \\
\hline 51 & 5 & 5-eil51 & 1 & 12 & 13 \\
\hline 51 & 10 & 10 -eil51 & 1 & 74 & 70 \\
\hline 51 & 15 & 15-eil51 & 1 & 2 & 1 \\
\hline 52 & 5 & 5 -berlin52 & 1 & 202 & 152 \\
\hline 52 & 10 & 10-berlin52 & 1 & 89 & 56 \\
\hline 52 & 15 & 15-berlin52 & 1 & 76 & 91 \\
\hline 70 & 15 & 15 -st70 & 1 & 884 & 605 \\
\hline 76 & 5 & 5-eil76 & 1 & 84 & 91 \\
\hline 76 & 10 & 10 -eil76 & 1 & 254 & 188 \\
\hline 76 & 15 & 15 -eil76 & 1 & 50 & 35 \\
\hline 76 & 5 & 5-pr76 & 1 & 99 & 301 \\
\hline 76 & 10 & $10-$ pr76 & 1 & 238 & 286 \\
\hline 76 & 15 & 15 -pr76 & 1 & 262 & 163 \\
\hline 99 & 10 & 10-rat99 & 1 & 651 & 800 \\
\hline 99 & 25 & 25-rat99 & 1 & 351 & 343 \\
\hline 99 & 50 & 50-rat99 & 1 & 2798 & 2305 \\
\hline 100 & 25 & 25-kroA100 & 1 & 3514 & 367 \\
\hline 100 & 50 & 50-kroA 100 & 1 & 948 & 543 \\
\hline 100 & 50 & $50-$ kroB100 & 1 & 2579 & 704 \\
\hline 101 & 25 & 25 -eil101 & 1 & 709 & 150 \\
\hline 101 & 50 & 50-eil101 & 1 & 275 & 357 \\
\hline 105 & 50 & $50-\operatorname{lin} 105$ & 1 & 1577 & 2801 \\
\hline \multicolumn{4}{|c|}{ tempo médio (tipo 1 ) (s) } & 714,91 & 473,73 \\
\hline 30 & 5 & i-30-5-17 & 5 & 1 & 1 \\
\hline 30 & 7 & i- $30-7-17$ & 5 & 3 & 2 \\
\hline 30 & 10 & $\mathrm{i}-30-10-17$ & 5 & 1 & 1 \\
\hline 45 & 5 & i- $45-5-18$ & 5 & 15 & 8 \\
\hline 45 & 7 & i- $45-7-18$ & 5 & 81 & 115 \\
\hline 45 & 10 & $\mathrm{i}-45-10-18$ & 5 & 60 & 99 \\
\hline 60 & 5 & i- $60-5-21$ & 5 & 527 & 829 \\
\hline 60 & 7 & i- $60-7-21$ & 5 & 711 & 176 \\
\hline 60 & 10 & $\mathrm{i}-60-10-21$ & 5 & 643 & 1220 \\
\hline 65 & 5 & i- $65-5-21$ & 5 & 1112 & 535 \\
\hline 65 & 10 & i- $65-10-21$ & 5 & 118 & 143 \\
\hline 70 & 5 & i- $70-5-21$ & 5 & 1994 & 250 \\
\hline 70 & 10 & i- $70-10-21$ & 5 & 701 & 2446 \\
\hline \multicolumn{4}{|c|}{ tempo médio (tipo 5) (s) } & 459,00 & 448,08 \\
\hline 51 & 4 & 4-eil51-2x2 & 6 & 8 & 8 \\
\hline 51 & 9 & 9 -eil51-3x3 & 6 & 8 & 8 \\
\hline 51 & 12 & 12 -eil51-3x4 & 6 & 1 & 1 \\
\hline 51 & 16 & 16 -eil51-4x4 & 6 & 9 & 11 \\
\hline 51 & 20 & 20 -eil51-4x5 & 6 & 9 & 14 \\
\hline 51 & 25 & 25 -eil51-5x5 & 6 & 1 & 5 \\
\hline 52 & 4 & 4-berlin52-2x2 & 6 & 226 & 210 \\
\hline 52 & 6 & 6-berlin52-2x3 & 6 & 204 & 329 \\
\hline 52 & 8 & 8-berlin $52-2 \times 4$ & 6 & 343 & 488 \\
\hline 52 & 10 & 10-berlin52-2x5 & 6 & 840 & 375 \\
\hline 70 & 12 & 12 -st70-3x4 & 6 & 2960 & 2167 \\
\hline 70 & 20 & 20 -st70-4x5 & 6 & 545 & 1733 \\
\hline 76 & 4 & 4-eil76-2x2 & 6 & 110 & 280 \\
\hline 76 & 9 & 9 -eil76-3x3 & 6 & 204 & 165 \\
\hline 76 & 12 & 12 -eil76-3x4 & 6 & 65 & 45 \\
\hline 76 & 16 & 16 -eil $76-4 \times 4$ & 6 & 26 & 35 \\
\hline 76 & 20 & $20-$ eil $76-4 \times 5$ & 6 & 48 & 28 \\
\hline 76 & 25 & 25 -eil76-5x5 & 6 & 28 & 27 \\
\hline 76 & 4 & $4-\operatorname{pr} 76-2 \times 2$ & 6 & 3482 & 1751 \\
\hline 76 & 9 & $9-$-pr76-3x3 & 6 & 197 & 200 \\
\hline 76 & 12 & $12-\operatorname{pr} 76-3 \times 4$ & 6 & 34 & 42 \\
\hline 76 & 15 & $15-\operatorname{pr} 76-3 \times 5$ & 6 & 430 & 3465 \\
\hline 76 & 18 & 18-pr76-3x6 & 6 & 1019 & 931 \\
\hline 99 & 25 & 25 -rat99-5x5 & 6 & 1000 & 992 \\
\hline 99 & 42 & 42 -rat99-6x7 & 6 & 2521 & 2048 \\
\hline 100 & 30 & $30-\mathrm{kroB} 100-5 \mathrm{x} 6$ & 6 & 368 & 561 \\
\hline 101 & 9 & 9 -eil101-3x3 & 6 & 1441 & 703 \\
\hline 101 & 36 & 36 -eil101-6x6 & 6 & 522 & 858 \\
\hline \multicolumn{4}{|c|}{ tempo médio (tipo 6$)$ (s) } & 594,61 & 624,29 \\
\hline \multicolumn{4}{|c|}{ tempo médio total (s) } & 608,64 & 535,35 \\
\hline
\end{tabular}

Tabela 1: Tempos alcançados pelo CPLEX.

Das 22 instâncias do tipo 1, 14 obtiveram tempos computacionais menores quando utilizam a formulação que penaliza as arestas inter-grupos, comparados aos tempos computacionais na formulação que não penaliza. O tempo computacional médio quando não se penalizam as arestas ficou em 714,91s e quando se penalizam em 473,73s. Para as instâncias do tipo 5, seis obtiveram 
tempos computacionais menores quando utilizam a formulação que não penaliza as arestas em um total de 13, comparados aos tempos computacionais na formulação que penaliza. Dois tempos computacionais foram iguais em ambas formulações. O tempo computacional médio quando não se penalizam as arestas ficou em 459,00s e quando se penalizam em 448,08s. Os tempos médios para as instâncias do tipo 5 foram bem próximos.

\begin{tabular}{|c|c||c|c|c|l|}
\hline \hline \#nós & $\# V_{i}$ & Instância & Valor & gap (\%) & status \\
\hline 30 & 5 & $\mathrm{i}-30-5-17$ & 5193 & 0,01 & sol. ótima \\
\hline 30 & 7 & $\mathrm{i}-30-7-17$ & 7940 & 0,01 & sol. ótima \\
\hline 30 & 10 & $\mathrm{i}-30-10-17$ & 6772 & 0,00 & sol. ótima \\
\hline 45 & 5 & $\mathrm{i}-45-5-18$ & 6879 & 0,01 & sol. ótima \\
\hline 45 & 7 & $\mathrm{i}-45-7-18$ & 7478 & 0,01 & sol. ótima \\
\hline 45 & 10 & $\mathrm{i}-45-10-18$ & 8258 & 0,01 & sol. ótima \\
\hline 60 & 5 & $\mathrm{i}-60-5-21$ & 7824 & 0,01 & sol. ótima \\
\hline 60 & 7 & $\mathrm{i}-60-7-21$ & 8623 & 0,01 & sol. ótima \\
\hline 60 & 10 & $\mathrm{i}-60-10-21$ & 9317 & 0,01 & sol. ótima \\
\hline 65 & 5 & $\mathrm{i}-65-5-21$ & 7680 & 0,01 & sol. ótima \\
\hline 65 & 7 & $\mathrm{i}-65-7-21$ & 8732 & 0,10 & sol. encontrada \\
\hline 65 & 10 & $\mathrm{i}-65-10-21$ & 9740 & 0,01 & sol. ótima \\
\hline 70 & 5 & $\mathrm{i}-70-5-21$ & 7793 & 0,01 & sol. ótima \\
\hline 70 & 7 & $\mathrm{i}-70-7-21$ & 9013 & 0,07 & sol. encontrada \\
\hline 70 & 10 & $\mathrm{i}-70-10-21$ & 9746 & 0,01 & sol. ótima \\
\hline 75 & 5 & $\mathrm{i}-75-5-22$ & 7327 & 1,06 & sol. encontrada \\
\hline 75 & 10 & $\mathrm{i}-75-10-22$ & 9278 & 0,40 & sol. encontrada \\
\hline 90 & 5 & $\mathrm{i}-90-5-33$ & 6678 & 0,58 & sol. encontrada \\
\hline 90 & 10 & $\mathrm{i}-90-10-33$ & 8727 & 0,31 & sol. encontrada \\
\hline 120 & 5 & $\mathrm{i}-120-5-46$ & 7890 & 1,23 & sol. encontrada \\
\hline 120 & 10 & $\mathrm{i}-120-10-46$ & 9734 & 0,21 & sol. encontrada \\
\hline
\end{tabular}

Tabela 2: Valores da função objetivo para as instâncias do tipo 5

Nas instâncias do tipo 6, de um total de 28, 13 instâncias obtiveram tempos computacionais menores ao utilizar a formulação que não penaliza as arestas inter-grupos. Em três instâncias os tempos computacionais foram iguais. O tempo computacional médio quando não se penalizam as arestas ficou em 594,61s e quando se penalizam em 624,29s. Observa-se que para as instâncias do tipo 6 a penalização aplicada nas arestas inter-grupos não é uma estratégia promissora. Considerando todas as instâncias da Tabela 1, o tempo computacional médio total ficou em 608,64 s ao utilizar a formulação que não penaliza as arestas inter-grupos e de $\mathbf{5 3 5 , 3 5 s}$ quando se penalizam.

\begin{tabular}{|c|c|c|c|}
\hline \#nós & $\overline{Z \# V_{i}}$ & Instâncias & Valor \\
\hline 51 & 5 & 5-eil51 & 437 \\
\hline 51 & 10 & 10 -eil51 & 440 \\
\hline 51 & 15 & 15-eil51 & 437 \\
\hline 52 & 5 & 5-berlin52 & 7991 \\
\hline 52 & 10 & 10-berlin52 & 7896 \\
\hline 52 & $\overline{15}$ & 15 -berlin52 & 8049 \\
\hline 70 & 5 & 5 -st70 & 695 \\
\hline 70 & 10 & 10 -st70 & 691 \\
\hline 70 & 15 & 15 -st70 & 692 \\
\hline 76 & 5 & 5-eil76 & 559 \\
\hline 76 & 10 & 10 -eil76 & 561 \\
\hline 76 & 15 & 15-eil76 & 565 \\
\hline 76 & 5 & $5-\operatorname{pr} 76$ & 108590 \\
\hline 76 & 10 & $10-$ pr76 & 109538 \\
\hline 76 & 15 & $15-\operatorname{pr} 76$ & 110678 \\
\hline 99 & 10 & 10 -rat99 & 1238 \\
\hline 99 & 25 & 25-rat99 & 1269 \\
\hline 99 & 50 & 50 -rat99 & 1249 \\
\hline 100 & 25 & 25-kroA100 & 21917 \\
\hline 100 & 50 & $50-\mathrm{kroA} 100$ & 21453 \\
\hline 100 & $\overline{10}$ & $10-$ kroB100 & 22440 \\
\hline 100 & 50 & 50-kroB100 & 22355 \\
\hline 101 & $\overline{25}$ & 25 -eil101 & 663 \\
\hline 101 & 50 & 50-eil101 & 644 \\
\hline 105 & 25 & $25-\operatorname{lin} 105$ & 14438 \\
\hline 105 & 50 & $50-\operatorname{lin} 105$ & 14379 \\
\hline 105 & 75 & $75-\operatorname{lin} 105$ & 14521 \\
\hline
\end{tabular}

Tabela 3: Valores da função objetivo para as instâncias do tipo 1

Na Tabela 2 apresentam-se os resultados obtidos para as instâncias do tipo 5 geradas variando o número de vértices de 30 a 120 e o número de grupos de 5 a 10 vértices. Na Tabela 2 é mostrado na primeira coluna o numero de vértices, na segunda o número de grupos, na terceira a instância, na quarta o valor alcançado pelo CPLEX, na quinta o percentual do gap calculado pelo CPLEX e na última coluna o status da solução. Para a instância i-65-7-21 com 65 vértices, 
para a instância i-70-7-21 com 70 vértices e todas as instâncias contendo 75, 90 e 120 vértices não foram encontradas soluções ótimas. Para as demais, o CPLEX alcançou soluções ótimas. O tempo máximo estabelecido ao CPLEX para encontrar soluções ótimas ou não, foi de 7200 segundos. Na Tabela 3 apresentam-se as instâncias do tipo 1 com o número de vértices na faixa 51 a 105 e número de grupos variando entre cinco e 75 vértices. Na primeira coluna da Tabela 3 é mostrado o número de vértices, na segunda coluna o número de grupos, na terceira as instâncias e na quarta o valor obtido pelo CPLEX. Para todas instâncias o CPLEX atingiu soluções ótimas.

\begin{tabular}{|c|c|c|c|}
\hline \#nós & $\# V_{i}$ & Instâncias & Valor \\
\hline 51 & 4 & 4 -eil51-2x2 & 429 \\
\hline 51 & 9 & 9 -eil51-3x3 & 445 \\
\hline 51 & 12 & $12-$ eil $151-3 \times 4$ & 441 \\
\hline 51 & 16 & $16-$ eil $51-4 \times 4$ & 438 \\
\hline 51 & 20 & 20 -eil51-4x5 & 441 \\
\hline 51 & 25 & 25 -eil51-5x5 & 437 \\
\hline 52 & 4 & 4-berlin52-2x2 & 8232 \\
\hline 52 & 6 & 6 -berlin52-2x3 & 8236 \\
\hline 52 & 8 & 8 -berlin52-2x4 & 8412 \\
\hline 52 & 10 & 10-berlin52-2x5 & 9267 \\
\hline 70 & 6 & 6 -st70-2x3 & 706 \\
\hline 70 & 9 & 9 -st70-3x3 & 703 \\
\hline 70 & 12 & 12 -st70-3x4 & 701 \\
\hline 70 & 16 & 16 -st70-4x4 & 714 \\
\hline 70 & 20 & 20 -st70-4x5 & 718 \\
\hline 76 & 4 & 4 -eil76-2x2 & 557 \\
\hline 76 & 9 & 9-eil76-3x3 & 560 \\
\hline 76 & 12 & 12 -eil76-3x4 & 562 \\
\hline 76 & 16 & $16-$ eil76-4x4 & 562 \\
\hline 76 & 20 & $20-$ eil76-4x5 & 562 \\
\hline 76 & 25 & 25 -eil76-5x5 & 557 \\
\hline 76 & 4 & $4-\operatorname{pr} 76-2 \times 2$ & 112071 \\
\hline 76 & 6 & 6-pr76-2x3 & 112573 \\
\hline 76 & 9 & $9-\operatorname{pr} 76-3 \times 3$ & 109123 \\
\hline 76 & 12 & 12-pr76-3x4 & 109164 \\
\hline 76 & 15 & 15 -pr76-3x5 & 115960 \\
\hline 76 & 18 & $18-\operatorname{pr} 76-3 \times 6$ & 117406 \\
\hline 99 & 25 & 25 -rat99-5x5 & 1377 \\
\hline 99 & 42 & $42-$ rat99-6x7 & 1318 \\
\hline 100 & 28 & 28 -kroA $100-4 \times 7$ & 25372 \\
\hline 100 & 25 & 25 -kroB100-5x5 & 24626 \\
\hline 100 & 30 & $30-\mathrm{kroB} 100-5 \times 6$ & 25244 \\
\hline 101 & 9 & 9-eil101-3x3 & 659 \\
\hline 101 & 36 & 36-eil101-6x6 & 658 \\
\hline 105 & 2 & $2-\operatorname{lin} 105-2 \times 1$ & 14627 \\
\hline 105 & 16 & $16-\operatorname{lin} 105-4 \times 4$ & 16516 \\
\hline
\end{tabular}

Tabela 4: Valores da função objetivo para as instâncias do tipo 6

A Tabela 4 mostra as soluções ótimas alcançadas pelo CPLEX para as instâncias do tipo 6 . O número de vértices mostrado na primeira coluna da Tabela 4 variaram de 51 a 105 vértices e o número de grupos (segunda coluna) entre quatro e 42 vértices. Na terceira coluna desta tabela são mostradas as instâncias e na quarta coluna o valor obtido pelo CPLEX.

\begin{tabular}{|c|c|c|c|c|c|c|c|}
\hline \multirow{2}{*}{ Instâncias } & \multirow{2}{*}{ Id. } & \multirow{2}{*}{ \#nós } & \multirow{2}{*}{$\# V_{i}$} & \multirow{2}{*}{ Tipo } & \multicolumn{3}{|c|}{ CPLEX } \\
\hline & & & & & Valor & Lim. Inf. & $(\%)$ \\
\hline i-50-gil262 & $J_{1}$ & 262 & 50 & 1 & 135529 & 135374,68 & 0,11 \\
\hline $10-\operatorname{lin} 318$ & $J_{2}$ & 318 & 10 & 1 & 534640 & 526412,07 & 1,54 \\
\hline $10-$ pcb442 & $J_{3}$ & 442 & 10 & 1 & 547152 & 536478,33 & 1,95 \\
\hline C1k.0 & $J_{4}$ & 1000 & 10 & 2 & 134025123 & 131354923,50 & 1,99 \\
\hline C1k.1 & $J_{5}$ & 1000 & 10 & 2 & 130750874 & 128540131,50 & 1,69 \\
\hline C1k.2 & $J_{6}$ & 1000 & 10 & 2 & 144341485 & 141501445 & 1,97 \\
\hline $300-6$ & $J_{7}$ & 300 & 6 & 3 & 8969 & 8915,18 & 0,60 \\
\hline $400-6$ & $J_{8}$ & 400 & 6 & 3 & 9117 & 9021,51 & 1,05 \\
\hline $700-20$ & $J_{9}$ & 700 & 20 & 3 & 41638 & 41274,00 & 0,87 \\
\hline $200-4-h$ & $J_{10}$ & 200 & 4 & 4 & 63429 & 62244,84 & 1,87 \\
\hline $200-4-x 1$ & $J_{11}$ & 200 & 4 & 4 & 60797 & 60242,96 & 0,91 \\
\hline $600-8-z$ & $J_{12}$ & 600 & 8 & 4 & 132897 & 127901,75 & 3,76 \\
\hline $600-8-x 2$ & $J_{13}$ & 600 & 8 & 4 & 132228 & 127901,75 & 3,27 \\
\hline $300-5-108$ & $J_{14}$ & 300 & 5 & 5 & 68361 & 67128,93 & 1,80 \\
\hline $300-20-111$ & $J_{15}$ & 300 & 20 & 5 & 311286 & 308595,45 & 0,86 \\
\hline $500-15-306$ & $J_{16}$ & 500 & 15 & 5 & 196001 & 193522,8 & 1,26 \\
\hline $500-25-308$ & $J_{17}$ & 500 & 25 & 5 & 367586 & 364108,13 & 0,95 \\
\hline 25 -eil101 & $J_{18}$ & 101 & 25 & 6 & 23671 & 23668,63 & 0,01 \\
\hline $42-\mathrm{a} 280$ & $J_{19}$ & 280 & 42 & 6 & 130043 & 129560,53 & 0,37 \\
\hline $144-$ rat783 & $J_{20}$ & 783 & 144 & 6 & 916174 & 913715,52 & 0,27 \\
\hline & & & & & & $g a p_{l i}$ médio $=$ & 1,36 \\
\hline
\end{tabular}

Tabela 5: Instâncias com seus identificadores $\left(J_{k}\right)$, número de nós, número de grupos, tipo e valores alcançados pelo CPLEX com limite de duas horas.

Os testes computacionais, usando a formulação Miller/Chisman e Miller/Pen, realizados nas instâncias do tipo 1, 5 e 6 alcançaram valores ótimos num total de 76 instâncias. Somente em 
oito casos as instâncias não atingiram a solução ótima dentro do tempo estabelecido. Toda estas instâncias (Tabelas 2, 3 e 4) são de pequeno porte com até 120 vértices. Para os outros tipos de instâncias não foram realizados testes computacionais utilizando estas formulações, pois as instâncias do tipo 2, 3 e 4 contém acima de 1000, 300 e 200 vértices, respectivamente. Isto inviabiliza encontrar soluções ótimas para estes últimos tipos de instâncias usando o software CPLEX executado no hardware descrito acima. Para as instâncias do tipo 2, 3 e 4 foram realizados testes computacionais através do CPLEX utilizando-se o modelo de Miller/Pen para se encontrar bons limites inferiores para estas instâncias, com número maior e igual a 200 vértices. Estes testes serão mostrados a seguir, na Tabela 5, assim como os testes computacionais para obter limites inferiores para as instâncias de porte maior do tipo 1, 5 e 6 contendo número de vértices acima de 100. A Tabela 5 mostra as instâncias (coluna 1) com os seus identificadores (coluna 2), seguido do número de vértices (nós), número de grupos e tipo. Na Tabela 5 são mostradas as soluções, os limites inferiores gerados pelo software CPLEX e na última coluna mostra-se o percentual do gap $_{l i}(\%)$ entre valor da solução e limite inferior calculado da seguinte forma:

$$
\operatorname{gap}_{l i}=100 *\left(\frac{\text { best }-l i}{\text { best }+\epsilon}\right),
$$

onde, best é o melhor valor encontrado pelo CPLEX, $l i$ o limite inferior dado pelo CPLEX e $\epsilon$ igual $10^{-10}$. Devido às longas iterações ocorridas no CPLEX para a maioria das instâncias, um tempo limite de 7200 s foi determinado para sua execução. Para a instância $J_{18}$, a solução ótima foi encontrada pelo CPLEX em 442s. Todas as outras execuções utilizaram 7200s, sendo, portanto abortadas antes de se encontrar ou confirmar uma solução ótima.

\section{Conclusão}

Com o método exato proposto utilizando a formulação de Miller/Chisman foi possível encontrar soluções ótimas para diversas instâncias do PCVG e bons limites inferiores. O tempo computacional despendido pelo método exato foi relativamente baixo usando a formulação penalizada ou não-penalizada de Miller. Observamos que para algumas instâncias a formulação penalizada não é promissora. Concluímos desta forma que o método com formulação de Miller/Chisman para o PCVG tem bom desempenho computacional validando desta forma a metodologia utilizada.

\section{Referências}

[1] ANILY, S.; BRAMEL, J.; HERTZ, A. A 5/3-approximation Algorithm for the Clustered Traveling Salesman Tour and Path Problems. Operations Research Letters, v. 24, n. 1-2, p. 29-35, 1999.

[2] CHISMAN, J. A. The Clustered Traveling Salesman Problem. Computers 8 Operations Research, v. 2, n. 2, p. 115-119, 1975.

[3] CPLEX. ILOG CPLEX 11.2 User's Manual and Reference Manual, 2009. In:. ILOG S.A.: [s.n.], 2009. Disponível em: <http://www.ilog.com>. Acesso em: 06/01/2009.

[4] DANTZIG, G.; FUlKERSON, R.; JOHNSON, S. Solution of a Large-Scale Traveling-Salesman Problem. Journal of the Operations Research Society of America, v. 2, p. 393-410, 1954.

[5] DING, C.; CHENG, Y.; HE, M. Two-Level Genetic Algorithm for Clustered Traveling Salesman Problem with Application in Large-Scale TSPs. Tsinghua Science and Technology, v. 12, n. 4, p. 459-465, 2007.

[6] GUTTMANN-BECK, N.; HASSIN, R.; KHULLER, S.; RAGHAVACHARI, B. Approximation Algorithms with Bounded Performance Guarantees for the Clustered Traveling Salesman Problem. Algorithmica, v. 28, n. 4, p. 422-437, 2000.

[7] JONGENS, K.; VOLGENANT, T. The Symmetric Clustered Traveling Salesman Problem. European Journal of Operational Research, v. 19, n. 1, p. 68-75, 1985.

[8] LAPORTE, G.; POTVIN, J.-Y.; QUILlERET, F. A Tabu Search Heuristic using Genetic Diversification for the Clustered Traveling Salesman Problem. Journal of Heuristics, v. 2, n. 3, p. 187-200, 1996.

[9] MESTRIA, M. Métodos Heurísticos usando GRASP, Reconexão de Caminhos e Busca em Vizinhança Variável para o Problema do Caixeiro Viajante com Grupamentos. Revista Produção Online, Florianópolis, SC, v. 13, n. 3, p. 1002-1033, 2013.

[10] MILLER, C. E.; TUCKER, A. W.; ZEMLIN, R. A. Integer Programming Formulation of Traveling Salesman Problems. Journal of the ACM, ACM Press, Nova York, NY, EUA, v. 7, n. 4, p. 326-329, 1960.

[11] ORMAN, A.; WILLIAMS, H. A Survey of Different Integer Programming Formulations of the Travelling Salesman Problem. Relatório Técnico, n. LSEOR 04.67. Department of Operational Research, London School of Economics and Political Science, Londres, Grã Bretanha, 2004.

[12] POtVIN, J.-Y.; GUERTIN, F. The Clustered Traveling Salesman Problem: A Genetic Approach. In: OSMAN, I. H.; KELLY, J. (Ed.). Meta-heuristics: Theory $\&$ Applications. Norwell, MA, EUA: Kluwer Academic Plublishres, 1996. cap. 37, p. 619-631. 